\title{
Non-equilibrium transport and spin dynamics in single-molecule magnets
}

\author{
V. Moldoveanu ${ }^{\text {a }}$, I.V. Dinu ${ }^{\mathrm{a}}$, B. Tanatar ${ }^{\mathrm{b}, *}$ \\ ${ }^{a}$ National Institute of Materials Physics, PO Box MG-7, Bucharest-Magurele, Romania \\ ${ }^{\mathrm{b}}$ Department of Physics, Bilkent University, Bilkent, 06800 Ankara, Turkey
}

\section{A R T I C L E I N F O}

\section{Article history:}

Received 5 March 2015

Received in revised form 17 June 2015

Accepted 18 June 2015

Available online 19 June 2015

\section{Keywords:}

Single-molecule magnets

Time-dependent transport

Molecular spintronics

\begin{abstract}
A B S T R A C T
The time-dependent transport through single-molecule magnets (SMM) coupled to magnetic or non-magnetic electrodes is studied in the framework of the generalized Master equation (GME) method. We calculate the transient currents which develop when the molecule is smoothly coupled to the source and drain electrodes. The signature of the electrically induced magnetic switching on these transient currents is investigated. Our simulations show that the magnetic switching of the molecular spin can be read indirectly from the transient currents if one lead is magnetic and it is much faster if the leads have opposite spin polarizations. We identify effects of the transverse anisotropy on the dynamics of molecular states.
\end{abstract}

(c) 2015 Elsevier Ltd. All rights reserved.

\section{Introduction}

Magnetic clusters and single-molecule magnets (SMM) are optimally suited to host spin manipulation schemes, and may therefore provide important steps in organic spintronics [1,2]. Transport properties of individual SMMs weakly coupled to gold electrodes have already been studied in various experiments. It was systematically confirmed that these systems display Coulomb blockade, sequential tunneling or negative differential resistance $[3,4]$. Kondo features were also investigated $[5,6]$. Electronic spins tunneling through the orbitals interact via the exchange coupling with the localized molecular spin. This opens the way to electrical switching of molecular spins [7]. Notably, spin polarized STM tips [8] can be used to measure spin-polarized transport. Moreover, SMMs or adatoms whose contacts are switched on and off by varying the substrate-tip spacing $[9,10]$ or by bending break junctions [11] are currently being investigated. Transient currents arising when a molecular tail couples to an STM tip were also recorded [12].

On the theoretical side the magnetic interactions in SMMs are conveniently described by effective Hamiltonians while the transport properties were mostly investigated within the steady-state rate-equation approach. Timm and Elste [13,14] derived the differential conductance of SMM and emphasized different transport regimes displaying spin amplification and negative differential conductance. Misiorny and Barnaś [15,16] studied transport with ferromagnetic leads. Memristive properties of SMM were also investigated [17]. An important outcome of these studies is that the current induced magnetic switching (CIMS) becomes possible as the system overcomes the anisotropy barrier $D S_{z}^{2}$ through charge transfer, $D$ being the easy-axis anisotropy constant. In the absence of quantum tunneling of magnetization (QTM) the full

\footnotetext{
* Corresponding author.

E-mail address: tanatar@fen.bilkent.edu.tr (B. Tanatar).
} 
magnetic switching requires the transient occupation of intermediate (excited) molecular states whose total spin scans all half-integer and integer values in the range $[-S, S]$, where $S$ the molecular spin.

In this work we initiate a theoretical study of time-dependent transport properties of SMM, the main focus is on the signatures of magnetic switching in the transient currents and on the time needed to perform the full magnetic switching. The paper is organized as follows: Section 2 introduces the model and summarizes the GME method, the results are discussed in Section 3; we conclude in Section 4.

\section{Theory}

The transport through a molecular system is typically mediated by its unoccupied orbitals which are coupled to source and drain gold-plated electrodes. In this work we consider for simplicity that the current is only due to electrons tunneling to and from the lowest unoccupied molecular orbital (LUMO). The effective Hamiltonian describing a local (molecular) spin $S$ which interacts with an itinerant electronic spin reads as:

$$
H_{M}=\sum_{\sigma} \epsilon a_{\sigma}^{\dagger} a_{\sigma}+U \hat{n}_{\uparrow} \hat{n}_{\downarrow}-g \mu_{B} B \widehat{S}_{z}^{t}-J \hat{\mathbf{s}} \cdot \hat{\mathbf{S}}-D \widehat{S}_{z}^{2}+E\left(\widehat{S}_{x}^{2}-\widehat{S}_{y}^{2}\right),
$$

where $\epsilon$ is the (spin degenerate) orbital energy, $J$ is the exchange interaction, $D$ is the easy-axis magnetic anisotropy constant while $E$ accounts for the transverse anisotropy. $B$ is the magnetic field applied along the $z$-axis. The electron-electron interaction strength is denoted by $U$. $\hat{\mathbf{S}}$ and $\hat{\mathbf{s}}$ are the operators associated with the localized and orbital spin, respectively. $\widehat{S}_{z}^{t}=\widehat{S}_{z}+\hat{S}_{z}$ stands for the total spin operator along the $z$-axis. $a_{\sigma}^{\dagger}\left(a_{\sigma}\right)$ are creation (annihilation) operators for spin $\sigma$, while the spin number operator is $\hat{n}_{\sigma}=a_{\sigma}^{\dagger} a_{\sigma}$.

In the absence of the transverse anisotropy (i.e. for $E=0$ ) the eigenstates of $H_{M}$ are labeled by the eigenvalues $m$ of the total spin operator $\widehat{S}_{z}^{t}$ since $\left[\widehat{S}_{z}^{t}, \hat{\mathbf{s}} \cdot \hat{\mathbf{S}}\right]=0$. Note that $m \pm 1 / 2$ scans the values of molecular spin projection $S_{z}=-S, \ldots, S$. The one-particle states corresponding to $m \in[-S+1 / 2, S-1 / 2]$ and are given by:

$$
|1, m\rangle^{ \pm}=C_{m \downarrow}^{ \pm}|\downarrow, m+1 / 2\rangle+C_{m \uparrow}^{ \pm}|\uparrow, m-1 / 2\rangle,
$$

where the coefficients $C_{m \sigma^{ \pm}}$have an explicit form (see e.g. [13]). For $m= \pm S$ the states $|1, \pm S\rangle$ have a well defined molecular spin. We shall denote by $\left|0, S_{z}\right\rangle$ the 'empty' molecular state (EMS) with molecular spin $S_{z}$ and energy $E_{0, S_{z}}=-D S_{z}^{2}-g \mu_{B} B S_{z}$. Finally one has also a set of two-particle states $\left\{\left|2, S_{z}\right\rangle\right\}$. The transverse anisotropy term induces mixing of states $\left|0, S_{z}\right\rangle$ and $\left|0, S_{z}^{\prime}\right\rangle$ if $\left|S_{z}-S_{z}^{\prime}\right|=2$, since $\widehat{S}_{x}^{2}-\widehat{S}_{y}^{2}=1 / 2\left(\widehat{S}_{+}^{2}+\widehat{S}_{-}^{2}\right)$. Same selection rule holds for one-particle states. We denote the eigenvalues and eigenfunctions of $H_{M}$ by $\mathscr{E}_{i, v}$ and $\left|\varphi_{i, v}\right\rangle$, where $i=0,1,2$ is the number of electrons on the molecular orbital. The index $v$ corresponds to the ordering of the levels, namely $\mathscr{E}_{0,1}<\mathscr{E}_{0,2} \ldots$ and similarly for $\mathscr{E}_{1, v}$.

We remark that if $g \mu_{B} B \ll D$ the tunneling between 'empty' molecular states $|0, m\rangle$ can be neglected if $S$ is half-integer, as the selection rule is verified only for states whose energies are separated by a large gap (e.g. $E_{0,1 / 2}$ and $E_{0,-3 / 2}$ ). In contrast, the transverse anisotropy couples degenerate one-particle states with opposite total spins. The strongest mixing is expected for the pair $|1,1\rangle^{ \pm}$and $|1,-1\rangle^{ \pm}$as the off-diagonal matrix element ${ }^{ \pm}\left\langle 1,1\left|H_{M}\right| 1,-1\right\rangle^{ \pm}$is linear in $E$. If the ratio $E / D$ increases, mixing effects to second order in $E$ have to also be taken into account and one has the general decompositions

$$
\left|\varphi_{1, v}\right\rangle=\sum_{s=+,-} \sum_{m}\left(c_{v, m}^{s}|1, m\rangle^{s}+c_{v,-m}^{s}|1,-m\rangle^{s}\right), \quad\left|\varphi_{0, v}\right\rangle=\sum_{S_{z}} c_{v, S_{z}}\left|0, S_{z}\right\rangle
$$

where $m$ is a positive integer. It is not difficult to show that for a given $\left|\varphi_{1, v}\right\rangle$ the total spin numbers $m$ must have the same parity. The full Hamiltonian $H_{M}$ is diagonalized numerically. We find that if the ratio $E / D$ is small enough for each $\varphi_{1, v}$ there is a single state with total magnetic number $\mathbf{m}$ whose weight $\left|c_{v, \mathbf{m}}^{s}\right|^{2}$ in Eq. (3) is by far the most important. Under these conditions the total magnetic quantum number is an 'almost' good quantum number and can still be used to label the states of the full Hamiltonian $H_{M}$. Then we introduce the simplified notations $\left|\varphi_{1, v}\right\rangle \approx|1, \mathbf{m}\rangle^{s}:=\left|\varphi_{1, \mathbf{m}}^{s}\right\rangle$ and $\left|\varphi_{0, v}\right\rangle \approx\left|0, S_{z}\right\rangle$ for some $S_{z}$.

For simplicity we model the source and drain electrodes as non-interacting semi-infinite tight-binding chains $L$ and $R$. We shall denote by $q$ the momentum of an electron propagating along the leads and by $t_{L}$ the hopping energy. The energy dispersion relation on the leads is $\epsilon_{q \sigma}=2 t_{L} \cos q+\Delta_{\sigma}$, where $\Delta_{\sigma}$ is the rigid-band spin splitting. The spin polarization in the leads is introduced as $P^{\alpha}:=\left(N_{+}^{\alpha}-N_{-}^{\alpha}\right) /\left(N_{+}^{\alpha}+N_{-}^{\alpha}\right)$, where $N_{+}^{\alpha}\left(N_{-}^{\alpha}\right)$ is the spin density of states (DOS) for the majority (minority) electrons in the lead $\alpha$. We consider equal charge densities in the leads.

The bias applied across the molecule is defined as $\mathrm{eV}=\mu_{L}-\mu_{R}$, where $\mu_{l}$ is the chemical potential of the lead $l$. We introduce two switching functions $\chi_{l}(t)(l=L, R)$ in order to describe the time-dependent coupling between the molecule and the leads. The explicit form chosen here is $\chi_{l}(t)=1-\frac{2}{e^{\gamma t}+1}$ where the parameter $\gamma$ allows us to control the lead-molecule coupling to be slower or faster. The lead-molecule contact Hamiltonian is written as follows

$$
H_{T}(t)=\sum_{l=L, R} \sum_{\sigma} \int d q \chi_{l}(t)\left(V_{q \sigma}^{l} a_{\sigma}^{\dagger} a_{q_{l} \sigma}+h . c\right),
$$


where $V_{q \sigma}^{l}$ is the tunneling coefficient. Without loss of generality we assume that $V_{q \sigma}^{l}$ does not depend on $q$ and that $V_{q \uparrow}^{l}=V_{q \downarrow}^{l}:=V^{l}$.

The transport properties were investigated using the generalized Master equation approach [18]. Essentially the method provides the SMM's reduced density operator (RDO) $\rho$ defined as the partial trace of the full density operator $W(t)$ over the leads' degrees of freedom $\rho(t)=\operatorname{Tr}_{\mathscr{F}_{L}}\{\mathrm{~W}(\mathrm{t})\}$, where $\mathscr{F}_{L}$ is the Fock space of the non-interacting leads. We shall consider only the weak coupling regime so the cotunneling contribution to transport can be neglected. The sequential tunneling processes are described by the following equation for the RDO

$$
\frac{d \rho(t)}{d t}=-\frac{i}{\hbar}\left[H_{M}, \rho(t)\right]-\frac{1}{\hbar^{2}} \operatorname{Tr}_{\mathscr{F}_{L}}\left\{\left[H_{T}(t), \int_{0}^{t} d s U(t, s)\left[H_{T}(s), \rho(s) \rho_{L}\right] U(t, s)^{*}\right]\right\},
$$

where we introduced the "free" evolution operator of the disconnected system $U(t, s)=e^{-i\left(H_{M}+H_{L}\right)(t-s) / \hbar}\left(H_{L}\right.$ is the leads' Hamiltonian). The GME is solved numerically with respect to the fully interacting states of $H_{M}$ and we calculate the diagonal elements of the RDO, e.g. $P_{\left|\varphi_{1 \mathbf{m}}^{s}\right\rangle}=\left\langle\varphi_{1, \mathbf{m}}^{s}|\rho(t)| \varphi_{1, \mathbf{m}}^{s}\right\rangle$. The orbital occupation is derived in terms of the diagonal elements (populations) associated with the SMM states which span the space $\mathscr{F}_{M}$

$$
\langle N(t)\rangle=\operatorname{Tr}_{\mathscr{F}_{M}}\{\rho(t) \widehat{N}\} .
$$

Similarly one can calculate the total transient currents in the source $(L)$ and drain $(R)$ leads, spin occupation and spin currents. We do not give all the formal details here and refer to Ref. [18] for a full description of this formalism. It is straightforward to show that the continuity equation leads to $J_{L}(t)-J_{R}(t)=\sum_{i} \sum_{v_{i}}\left\langle\varphi_{i, v_{i}}|\dot{\rho}(t)| \varphi_{i, v_{i}}\right\rangle$. Then one can identify the transient currents by replacing in the RHS derivative of RDO from Eq. (5). It is easy to see that 'empty' molecular states $\left|0, S_{z}^{t}\right\rangle$ do not contribute to the transport.

\section{Numerical results and discussion}

Our simulation were performed for systems described by a molecular spin $S=5 / 2$. The initial state is assumed to be $\rho(t=0)=|0,5 / 2\rangle\langle 0,5 / 2|$ which can be selected by applying a small perpendicular magnetic field. The electron-electron repulsion is considered to be strong, i.e. $U \gg J$. In this case the chemical potential of the leads can be chosen such that the two-electron configurations $\left|\varphi_{2, v}\right\rangle$ are pushed above the bias window and do not contribute to transport.

In Fig. 1(a) we collect the spectral properties of the isolated SMM system for $S=5 / 2$ as a function of the dominant magnetic number $\mathbf{m}$. The ratio $E / D=1 / 25$ and $B=0.65 \mathrm{mT}$; for these parameters the weight of $\mathbf{m}$ in Eq. (3) is at least 0.8 for each state. Fig. 1(b) pictures some transitions from the initial EMS $|0, S\rangle$ to neighbor states. The tunneling leads to half-integer 'jumps' of the total magnetic number $m$.

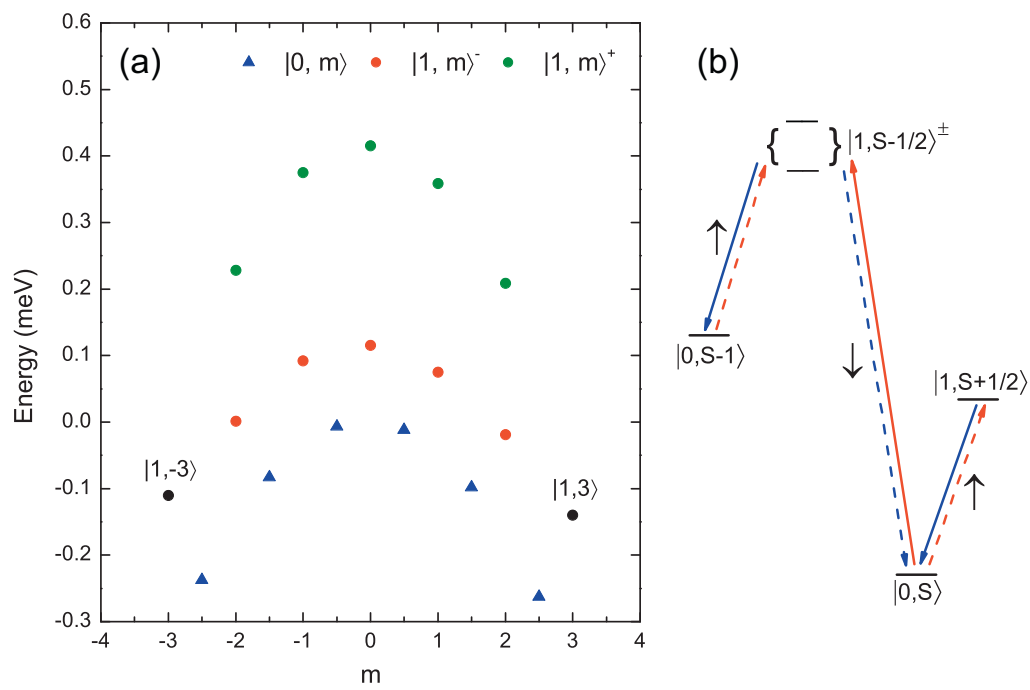

Fig. 1. (a) The spectrum of an $S=5 / 2 \mathrm{SMM}$ as a function of the dominant total magnetic quantum number $m$ (see Eq. (3)). The two-particle sector is not included as the corresponding states are not involved in transport. Other parameters: $\epsilon=0.25 \mathrm{meV}, J=0.1 \mathrm{meV}, U=1 \mathrm{meV}, g \mu_{B} B=0.65 \mathrm{mT}$, $D=0.04 \mathrm{meV}$ and $E / D=1 / 25$. (b) Magnetic transitions connecting the states $|0, S\rangle$ and $|0, S-1\rangle$ via back-and-forth tunneling with the left (L) and right (R) leads. Full red line: forward charging, full blue line: forward discharging, dashed red line: backward charging, dashed blue line: backward discharging. The black arrows mark the spin $\sigma$ involved in tunneling processes. (For interpretation of the references to color in this figure legend, the reader is referred to the web version of this article.) 
We identify 'forward' processes $m \rightarrow m-1 / 2$ (the full line arrows) which contribute to the magnetic switching $S \rightarrow-S$ and 'backward' processes $m \rightarrow m+1 / 2$ competing the total spin reversal (dashed lines). One can also distinguish between 'absorption' of spin-down electrons from the leads (charging of the molecular orbital along the transitions $|0, S\rangle \rightarrow|1, S-1 / 2\rangle^{ \pm}$) and tunneling of spin-up electrons from the molecular orbital (i.e. a depletion process associated to the transitions $|1, S-1 / 2\rangle^{ \pm} \rightarrow|0, S-1\rangle$ ). Similarly one defines charging and discharging 'backward' processes (see the dashed lines).

We studied the transient regime for three spin configurations of the leads. In the simplest setting both leads are non-magnetic (NM), that is $P^{L}=P^{R}=0$. A second configuration corresponds to a non-magnetic source electrode while the drain electrode carries only spin-up electrons, i.e. $P^{L}=0, P^{R}=1$ and $N_{+}^{R}=N_{\uparrow}^{R}$; we call this setting normal-ferromagnetic (NF). Finally, in the antiparallel (AP) configuration both electrodes are ferromagnetic and have opposite magnetizations, namely the left lead carries spin-down electrons and the right lead spin-up electrons.

In Fig. 2(a) we show the transient current $J_{L}$ in the source (left) electrode for these configurations. The current through the drain (right) electrode $J_{R}$ was also calculated and coincides with $J_{L}$ in the stationary regime. In all settings $J_{L}$ develops a sharp peak at short times which corresponds to the fast charging of the molecular orbital when the contact to the leads is established. One can easily notice qualitative differences between the three configurations. In the non-magnetic (NM) case the steady-state establishes quite rapidly around $t=0.25 \mathrm{~ns}$. The total charge accumulated on the orbital in the stationary regime is $Q \sim 0.67$ with equal spin occupations (see also Fig. 3(a) below). In the normal-ferromagnetic (NF) configuration $J_{L}$ decreases slowly to zero, while the antiparallel (AP) setting leads to a vanishing current much earlier, at $t \sim 3 \mathrm{~ns}$. The suppression of transport coincides with the spin-down filling of the molecular orbital (see also Fig. 3(b) and (c)). We have checked that in the NF and AP configurations the system ends up in a single state, namely $\left|\varphi_{1,-3}\right\rangle=|\downarrow,-5 / 2\rangle$. No current can flow as long as the right electrode is spin-down polarized.

A natural question is whether the transient current allows one to extract some information on the all-electrical magnetic switching of the molecular spin from $|0,5 / 2\rangle \rightarrow|0,-5 / 2\rangle$. Fig. 2(b) shows the average total spin $\left\langle S_{z}^{t}\right\rangle$ in the NM, NF and AP transport regimes. The non-magnetic setting does not provide any hints on the molecular spin dynamics as $\left\langle S_{z}^{t}\right\rangle$ varies even when $J_{L}$ is stationary. Note also that $\left\langle S_{z}^{t}\right\rangle$ relaxes slowly and, more importantly, settles down to small positive value. When analyzing the non-vanishing populations in the steady state we find equal occupation for states with opposite total spin $m$ (e.g. $\left|\varphi_{1, \pm \mathbf{3}}\right\rangle$ and $\left|\varphi_{1, \pm \mathbf{2}}^{ \pm}\right\rangle$), which means that in this configuration the magnetic switching cannot pe achieved because the 'forward' and 'backward' tunneling processes coexists. Note that a complete magnetic switching requires a large (ideally equal to 1$)$ population for the leftmost state $\left|\varphi_{1,-3}\right\rangle$. This condition cannot be achieved unless $N_{\uparrow}$ is very small.

The magnetic transition is enhanced in the NF configuration and $\left\langle S_{z}^{t}\right\rangle$ settles down to -3 around $t \sim 5.5 \mathrm{~ns}$. We checked again that in the steady-state the single non-vanishing population is $P_{\varphi_{(1,-3)}}=1$; a similar feature is obtained in the AP setting. A clear connection between the transient current and the full magnetic switching exists both in the NF and AP configuration. The transient current vanishes precisely when $\left\langle S_{z}^{t}\right\rangle$ is completely reversed. The magnetic switching operation is much faster in the AP configuration. Otherwise stated, the magnetic switching slows down if the 'rightmost' state $\left|\varphi_{1,3}\right\rangle$ is significantly charged in the transient regime.
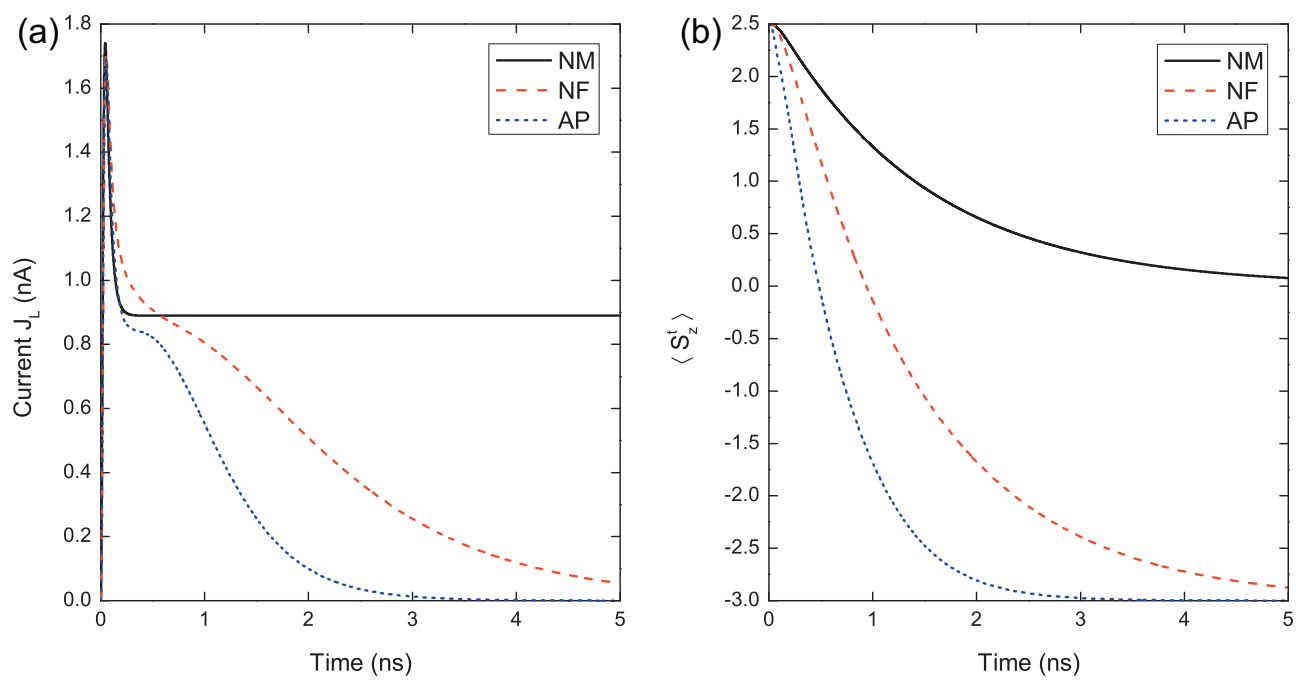

Fig. 2. (a) Transient current in the source electrode for different spin polarizations of the leads. (b) The average total spin $\left\langle S_{z}^{t}\right\rangle$. More discussion is given in the text. $\mu_{L}=1 \mathrm{meV}, \mu_{R}=-1 \mathrm{meV} . V^{L}=V^{R}=0.045 \mathrm{meV}$. Other parameters are as in Fig. 1(a). 

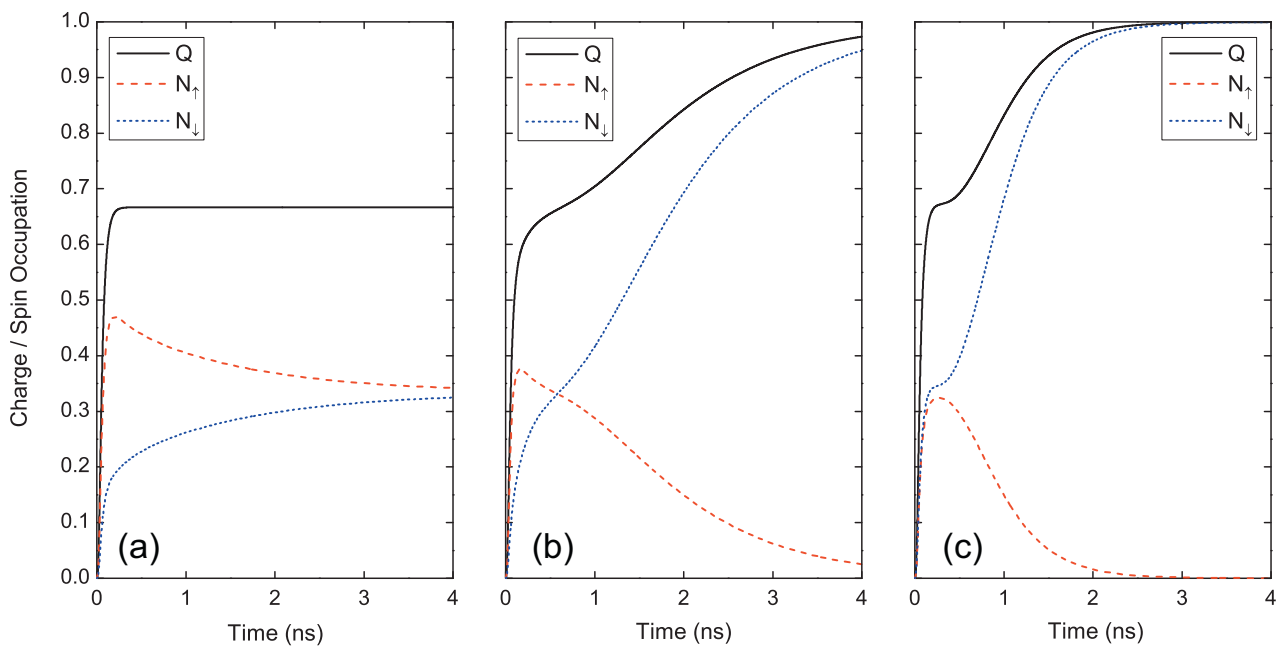

Fig. 3. Charge and spin occupations of the LUMO for different spin polarizations of the leads. (a) Non-magnetic (NM) leads, (b) normal-ferromagnetic (NF) setting, (c) antiparallel (AP) configuration. The parameters are as in Fig. 2.

Fig. 3 shows the evolution of the total charge $Q$ and spin occupation numbers $N_{\sigma}$. In Fig. 3(a) and (b) the spin-up occupation has the fastest dynamics in the 'charging' regime $(t<0.25 \mathrm{~ns})$. This is due to the fact that spin-up electrons can be added on $\left|\varphi_{1,2}^{ \pm}\right\rangle$and $\left|\varphi_{1,3}\right\rangle$, while spin-down electrons are not allowed on the latter state. However, since the spin-down tunneling to the right lead is forbidden $N_{\downarrow}$ continuosly increases in the NF and AP configurations. In contrast, $N_{\uparrow}$ vanishes in the steady state as the SMM settles down to the state $\varphi_{|1,-3|}$. By looking at the SMM spectrum along with the selection rules for tunneling one would expect that the states are being activated (i.e. populated) according to the sequence $\left|\varphi_{1,2}^{ \pm}\right\rangle \rightarrow|0,3 / 2\rangle \rightarrow\left|\varphi_{1,1}^{ \pm}\right\rangle \rightarrow|0,1 / 2\rangle$, etc. Surprisingly, the dynamics of the corresponding populations in the NF setting shows (see Fig. 4(a)) that $P_{\varphi_{11}^{-}-1 \mathbf{1}}$ emerges simultaneously with $P_{\varphi_{1,1}^{-}, 1}$ and earlier than $P_{\varphi_{1,0}^{-} .}$. This suggest an underbarrier tunneling due to the transverse anisotropy $E$. For $E / D=1 / 25$ one finds indeed that the states $\varphi_{|1,-1\rangle}^{ \pm}$have a minoritary component $|1,1\rangle^{ \pm}$, that is $\left|c_{1,1}^{ \pm}\right|^{2} \sim 0.18$. As a consequence of this mixing the system undergoes transitions from the EMS $|0,3 / 2\rangle$ not only to $\varphi_{|1, \mathbf{1}\rangle}^{ \pm}$but also to $\varphi_{\{1,-1}^{ \pm}$. In order to confirm this picture we performed the same simulation for $E / D=1 / 150$. From Fig. 4(b) we notice that in this case $P_{\varphi_{1,0}^{-}}$rises before $P_{\varphi_{(1,-1)}^{-}}$because the quantum tunneling of magnetization is suppressed at small $E$. A similar behavior is found for ' + ' states.
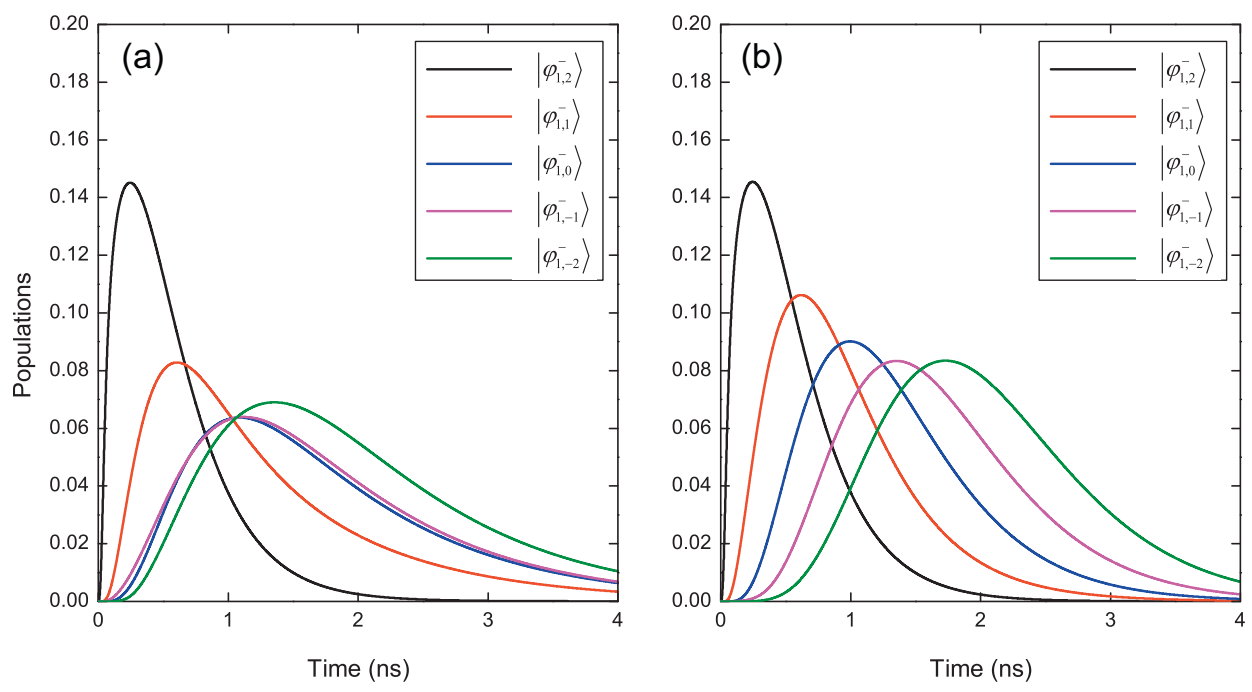

Fig. 4. Transverse anisotropy effects on the transient occupation of one-particle states in the NF configuration. (a) $E / D=1 / 25-\left|\varphi_{1,1}\right\rangle^{-}$and $\left|\varphi_{1,-1}\right\rangle^{-}$are simultaneously populated. (b) $E / D=1 / 150-\left|\varphi_{1,-1}\right\rangle^{-}$populates later than $\left|\varphi_{1,0}\right\rangle^{-}$. Other parameters are as in Fig. 2. 


\section{Conclusions}

We have implemented the generalized Master equation (GME) method for transport through a single-magnetic molecule coupled to ferromagnetic or nonmagnetic leads and discussed the time-dependent spin transport and molecular spin dynamics. For non-magnetic electrodes the evolution of the total spin $S_{z}^{t}$ cannot be traced back from the transient current. The onset of a steady state transport regime implies neither that all projections of the total spin $S_{z}^{t}$ have been spanned nor that the spin reversal is accomplished. In contrast, the magnetic switching can be read from the transient current if one of the leads is magnetic. In the NF and AP configurations the steady state current vanishes because the orbital is spin-down polarized from the left lead and the drain lead allows only spin-up tunneling. The full magnetic switching coincides with the onset of the steady-state.

Our results show that by measuring the transient current in the antiparallel configuration one can extract the time needed for the system to experience all intermediate molecular state between $|0, S\rangle$ and $|0,-S\rangle$. The analysis of transient currents within the GME method is a meaningful and relevant generalization of previous theoretical work and should provide important insight into molecular spin switching protocols.

\section{Acknowledgements}

V.M. and I.V.D. acknowledge financial support from PNCDI2 program (grant PN-II-ID-PCE-2011-3-0091) and from Grant No. 45N/2009. V.M., I.V.D. and B.T. acknowledge financial support from ANCS-TUBITAK Bilateral Programme COBIL 603/2013 and 112T619. B.T. also thanks TUBA for support.

\section{References}

[1] L. Bogani, W. Wernsdorfer, Molecular spintronics using single-molecule magnets, Nat. Mater. 7 (2008) $179-186$.

[2] S. Sanvito, Molecular spintronics, Chem. Soc. Rev. 40 (2011) 3336-3355.

[3] H.B. Heersche, Z. de Groot, J.A. Folk, H.S.J. van der Zant, C. Romeike, M.R. Wegewijs, L. Zobbi, D. Barreca, E. Tondello, A. Cornia, Electron transport through single $\mathrm{mn}_{12}$ molecular magnets, Phys. Rev. Lett. 96 (2006) 206801.

[4] M.-H. Jo, J.E. Grose, K. Baheti, M.M. Deshmukh, J.J. Sokol, E.M. Rumberger, D.N. Hendrickson, J.R. Long, H. Park, D.C. Ralph, Signatures of molecular magnetism in single-molecule transport spectroscopy, Nano Lett. 6 (9) (2006) 2014-2020.

[5] J.J. Parks, A.R. Champagne, T.A. Costi, W.W. Shum, A.N. Pasupathy, E. Neuscamman, S. Flores-Torres, P.S. Cornaglia, A.A. Aligia, C.A. Balseiro, G.K.-L. Chan, H.D. Abrua, D.C. Ralph, Mechanical control of spin states in spin-1 molecules and the underscreened kondo effect, Science 3284 (598) (2010) 13701373.

[6] T. Komeda, H. Isshiki, J. Liu, Y.-F. Zhang, N. Lorente, K. Katoh, B.K. Breedlove, M. Yamashita, Observation and electric current control of a local spin in a single-molecule magnet, Nat. Commun. 2 (217) (2011) 1-7.

[7] A.S. Zyazin, J.W.G. van den Berg, E.A. Osorio, H.S.J. van der Zant, N.P. Konstantinidis, M. Leijnse, M.R. Wegewijs, F. May, W. Hofstetter, C. Danieli, A. Cornia, Electric field controlled magnetic anisotropy in a single molecule, Nano Lett. 10 (9) (2010) 3307-3311.

[8] R. Wiesendanger, Spin mapping at the nanoscale and atomic scale, Rev. Mod. Phys. 81 (2009) 1495-1550.

[9] A. Kumar, R. Heimbuch, B. Poelsema, H.J.W. Zandvliet, Controlled transport through a single molecule, J. Phys.: Condens. Matter 24 (8) (2012) 082201.

[10] K. Sotthewes, R. Heimbuch, H.J.W. Zandvliet, Manipulating transport through a single-molecule junction, J. Chem. Phys. 139 (2013) 214709.

[11] S. Ballmann, H.B. Weber, An electrostatic gate for mechanically controlled single-molecule junctions, New J. Phys. 14 (12) (2012) 123028.

[12] D. Kockmann, B. Poelsema, H.J.W. Zandvliet, Transport through a single octanethiol molecule, Nano Lett. 9 (3) (2009) $1147-1151$.

[13] C. Timm, F. Elste, Spin amplification, reading, and writing in transport through anisotropic magnetic molecules, Phys. Rev. B 73 (2006) 235304.

[14] F. Elste, C. Timm, Transport through anisotropic magnetic molecules with partially ferromagnetic leads: spin-charge conversion and negative differential conductance, Phys. Rev. B 73 (2006) 235305.

[15] M. Misiorny, J. Barna, Spin polarized transport through a single-molecule magnet: current-induced magnetic switching, Phys. Rev. B 76 (2007) 054448.

[16] M. Misiorny, J. Barna, Magnetic switching of a single molecular magnet due to spin-polarized current, Phys. Rev. B 75 (2007) 134425.

[17] C. Timm, M. Di Ventra, Memristive properties of single-molecule magnets, Phys. Rev. B 86 (2012) 104427.

[18] V. Moldoveanu, A. Manolescu, C.-S. Tang, V. Gudmundsson, Coulomb interaction and transient charging of excited states in open nanosystems, Phys. Rev. B 81 (2010) 155442. 